\title{
LIPÍDIOS COMO INDICADORES DE MUDANÇAS CLIMÁTICAS NO PASSADO 1: BIOMARCADORES MARINHOS
}

Luiz A. S. Madureira

Departamento de Química - Campus Universitário Trindade - UFSC - 88040-900 - Florianópolis - SC

Recebido em 21/3/96; aceito em 27/6/96

\begin{abstract}
LIPIDS AS INDICATORS OF PALEOCLIMATIC CHANGES 1: MARINE BIOMARKERS. The distribution and abundance of specific lipid biomarkers in marine sediments can play an important rule for the reconstruction of past climatic changes. Here, it is shown the use of a widely known paleo Sea Surface Temperature (SST) proxy based on the unsaturation ratio of a suite of long-chain $C_{37}$ alkenones: the alkenone unsaturation index, $U^{K}{ }_{37}$. These compounds are biosynthesised by only a restricted group of prymnesiophyte algae, mostly notably the coccolithophorid Emiliania huxleyi. To ilustrate the importance of the $\mathrm{U}^{\mathrm{K}}{ }_{37}$, index as a molecular tool, the correlation between the SST signal derived from the $\mathrm{U}^{\mathrm{K}}{ }_{37}$, and other paleoclimatic records examined in a core from the North Atlantic is discussed.
\end{abstract}

Keywords: alkenones; marine sediments; North Atlantic.

\section{INTRODUÇÃO}

Há cerca de dezoito anos, uma série de metil- e etil-alquenonas de cadeias longas, di- e tri-insaturadas $\left(\mathrm{C}_{37}-\mathrm{C}_{39}\right.$ alcadienonas e alcatrienonas), foram identificadas em sedimentos da Cordilheira de Walvis (DSDP Leg $\left.40^{1}\right)^{2}$. Coincidentemente, com a tentativa da determinação da estrutura desses compostos através da espectrometria de massas por de Leeuw e colaboradores $^{3}$ em 1980, essas alquenonas, além de uma série de alquenoatos de alquila derivados do ácido hexatriacontanóico, foram também identificados no cocolitoforídeo Emiliania huxleyi ${ }^{4}$, uma espécie de alga marinha encontrada em várias regiões nos oceanos, bem como em outros sedimentos oceâni$\cos ^{3,5}$ (Tabela 1 e Fig. 1). Nos anos seguintes, houve um grande aumento no número de sedimentos marinhos imaturos onde esses compostos eram reconhecidos, extendendo-se do Quaternário $\left(1,8 \times 10^{6}\right.$ anos) até o Cretáceo $\left(65 \times 10^{6} \text { anos }\right)^{6-10}$. Há ainda trabalhos que reportam a presença dessas alquenonas em sedimentos lacustres do Quaternário ${ }^{11^{3}}$.

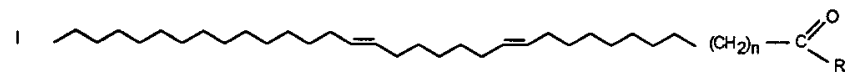

II
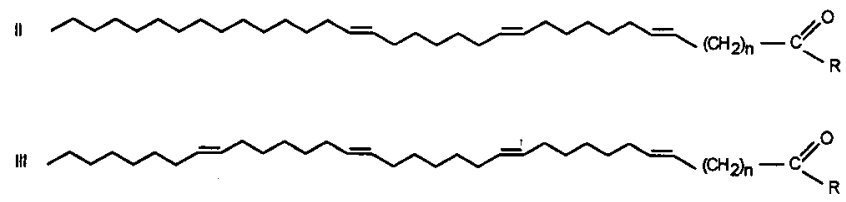

Figura 1. Estruturas das alquenonas e dos alquenoatos de alquila. Ver os nomes e os valores de $n$ na Tabela 1.

Como em toda abordagem da geoquímica orgânica, tornouse necessário então avaliar a distribuição desses compostos em organismos vivos a fim de se inferir qual ou quais espécies que possivelmente poderiam ter contribuído para a formação do ambiente sedimentar onde tais compostos são encontrados.

No princípio, as alquenonas e os ésteres, assim como alguns esteróis, foram investigados em várias espécies de alguns gêneros de algas da Classe Prymnesiophyceae, com o objetivo de se estabelecer não somente uma correlação fonte-origem, mas também obter informações quimiotaxonômicas, isto é, investigar a composição química de algas de diferentes espécies de uma mesma Classe ${ }^{6,12}$. Em seus estudos usando culturas de laboratório, Marlowe observou a presença desses compostos em quatro espécies de primnesiofita (do inglês prymnesiophyte), além, é claro, do abundante cocolitoforídeo marinho Emiliania huxleyi.

\section{VARIAÇÕES COM A TEMPERATURA}

As alquenonas começaram a despertar o interesse da comunidade geoquímica quando Marlowe ${ }^{6}$ demonstrou que o $p a$ drão de insaturação dessas alquenonas variava em resposta à temperatura na qual as algas eram cultivadas. À medida que a temperatura de cultivo era aumentada, diminuia o número de insaturações, isto é, o número médio de alquenonas tri- e tetrainsaturadas em relação às di-insaturadas. Tal comportamento na mudança do padrão de insaturação com a temperatura já era conhecido para outras classes de lipídios, notadamente ácidos carboxílicos e ésteres de cadeias longas ${ }^{13,14}$. De fato, organismos aquáticos podem biossintetizar um certo conjunto de lipídios que os capacitem a viver sob determinadas condições ${ }^{15}$. Variações na composição molecular da camada de lipídios ajudam, por exemplo, a manter a fluidez das membranas, seja através de alterações no comprimento das cadeias ou no $p a$ drão de insaturação. Entretanto, no caso particular das alquenonas e dos alquenoatos de alquila, ainda não foram comprovadas as suas funções nas algas.

Até 1986, já haviam sido publicados diversos trabalhos que relatavam a presença das alquenonas e, em alguns casos, dos alquenoatos de alquila em sedimentos marinhos do Quaternário coletados durante várias expedições oceanográficas, principalmente nas do programa DSDP. A idéia de se usar o padrão de insaturação dessas alquenonas como uma medida da variação da temperatura das águas superficiais nos oceanos, originou-se a partir da observação de que o número de insaturações variava de acordo com a região de onde a amostra, sedimentar ou particulado em suspensão, era coletada. O reconhecimento de que sedimentos provenientes de regiões sob águas mais quentes continham menor proporção de alcatrienonas e alcatetraenonas em relação às alcadienonas quando comparados com os sedimentos de regiões sob águas mais frias, levou Brassell e colaboradores ${ }^{8}$ a considerar o uso desses biomarcadores para estudos de paleotemperaturas. De fato, em 1986, esses autores mostraram a existência de uma relação entre a variação no padrão de insaturação das alquenonas em sedimentos marinhos 
Tabela 1. Nomes das alquenonas e dos alquenoatos de alquila identificados em algas e sedimentos marinhos ${ }^{6}$. A numeração na primeira coluna da tabela corresponde aos picos assinalados no cromatograma da Fig. 2, com os respectivos nomes e abreviaturas

\begin{tabular}{|c|c|c|c|c|}
\hline $\begin{array}{l}\text { Números } \\
\text { dos picos na } \\
\text { Figura } 2\end{array}$ & Nome $^{\mathrm{a}}$ e abreviatura ${ }^{\mathrm{b}}$ & $\mathrm{n}$ & $\begin{array}{l}\text { Estrutura } \\
\quad(\text { ver } \\
\text { Figura 1) }\end{array}$ & $\mathrm{R}$ \\
\hline 1 & heptatriaconta-8E,15E,22E,29E-tetraen-2-ona $\left(\mathrm{C}_{37: 4 \mathrm{Me}}\right)$ & 5 & III & $\mathrm{CH}_{3}$ \\
\hline 2 & heptatriaconta-8E,15E,22E-trien-2-ona $\left(\mathrm{C}_{37: 3 \mathrm{Me}}\right)$ & 5 & II & $\mathrm{CH}_{3}$ \\
\hline 3 & hexatriaconta-7E,14E,21E-trienoato de metila $\left(\mathrm{C}_{36: 3 \mathrm{FAME}}\right)$ & 5 & II & $\mathrm{OCH}_{3}$ \\
\hline 4 & heptatriaconta-15E,22E-dien-2-ona $\left(\mathrm{C}_{37: 2 \mathrm{Me}}\right)$ & 5 & I & $\mathrm{CH}_{3}$ \\
\hline 5 & hexatriaconta-14E,21E-dienoato de metila $\left(\mathrm{C}_{36: 2 \mathrm{FAME}}\right)$ & 5 & II & $\mathrm{OCH}_{3}$ \\
\hline 6 & octatriaconta-9E,16E,23E-trien-3-ona $\left(\mathrm{C}_{38: 3 \mathrm{Et}}\right)$ & 5 & II & $\mathrm{C}_{2} \mathrm{H}_{5}$ \\
\hline 7 & octatriaconta-9E,16E,23E-trien-2-ona $\left(\mathrm{C}_{38: 3 \mathrm{Me}}\right)$ & 6 & II & $\mathrm{CH}_{3}$ \\
\hline 8 & octatriaconta-16E,23E-dien-3-ona $\left(\mathrm{C}_{38: 2 \mathrm{Et}}\right)$ & 5 & I & $\mathrm{C}_{2} \mathrm{H}_{5}$ \\
\hline 9 & octatriaconta-16E,23E-dien-2-ona $\left(\mathrm{C}_{38: 2 \mathrm{Me}}\right)$ & 6 & I & $\mathrm{CH}_{3}$ \\
\hline 10 & nonatriaconta-10E,17E,24E-trien-2-ona $\left(\mathrm{C}_{39: 3 \mathrm{Me}}\right)$ & 7 & II & $\mathrm{CH}_{3}$ \\
\hline- & octatriaconta-9E,16E,23E,30E-tetraen-3-ona & 5 & III & $\mathrm{C}_{2} \mathrm{H}_{5}$ \\
\hline- & octatriaconta-9E, 16E,23E,30E-tetraen-2-ona & 6 & III & $\mathrm{CH}_{3}$ \\
\hline- & nonatriaconta-10E,17E,24E-trien-3-ona & 6 & II & $\mathrm{C}_{2} \mathrm{H}_{5}$ \\
\hline- & nonatriaconta-17E,24E-dien-3-ona & 6 & I & $\mathrm{C}_{2} \mathrm{H}_{5}$ \\
\hline- & hexatriaconta-7E,14E,21E,28E-tetraenoato de etila ${ }^{c}$ & 5 & III & $\mathrm{OC}_{2} \mathrm{H}_{5}$ \\
\hline- & hexatriaconta-7E, 14E,21E-trienoato de etila ${ }^{c}$ & 5 & II & $\mathrm{OC}_{2} \mathrm{H}_{5}$ \\
\hline- & hexatriaconta-14E,21E-dienoato de etila & 5 & I & $\mathrm{OC}_{2} \mathrm{H}_{5}$ \\
\hline
\end{tabular}

${ }^{a}$ As configurações das alquenonas $C_{38}$ e $C_{39}$ e as dos ésteres $C_{36}$ foram inferidas por analogia com as configurações determinadas para as alquenonas $\mathrm{C}_{37}$, conforme descrito por Rechka e Maxwell ${ }^{18}$.

${ }^{\mathrm{b}}$ A abreviatura entre parênteses ao lado de cada nome representa o número de átomos de carbono : número de ligações duplas e o substituinte alquila.

${ }^{\mathrm{c}}$ Compostos detectados somente em algas ${ }^{6}$.

de uma ampla variedade de latitudes e a temperatura da superfície aquática habitada pelas algas. Assim, aparecia pela primeira vez um índice baseado na razão das alquenonas $\mathrm{C}_{37}$ (Eq. 1) denominado de índice de insaturação, $\mathrm{U}^{\mathrm{K}}{ }_{37}$ (do inglês Unsaturated Ketones $)^{8}$ :

$$
U_{37}^{K}=\frac{\left[C_{37: 2 M e}\right]-\left[C_{37: 4 M e}\right]}{\left[C_{37: 2 M e}+C_{37: 3 M e}+C_{37: 4 M e}\right]}
$$

onde, $\mathrm{C}_{37: 2 \mathrm{Me}}, \mathrm{C}_{37: 3 \mathrm{Me}}$ e $\mathrm{C}_{37: 4 \mathrm{Me}}$ correspondem às $\mathrm{C}_{37}$ metil-alquenonas com duas, três e quatro insaturações, respectivamente.

Para uma grande parte das amostras sedimentares dos oceanos, o índice $\mathrm{U}^{\mathrm{K}}{ }_{37}$ pode ser simplificado devido à ausência do composto $\mathrm{C}_{37: 4 \mathrm{Me}}$ :

$$
U_{37}^{K^{\prime}}=\frac{\left[C_{37: 2 M e}\right]}{\left[C_{37: 2 M e}+C_{37: 3 M e}\right]}
$$

Para os sedimentos marinhos do Quaternário Recente $(0,75$ x $10^{6}$ anos), esses autores mostraram que os maiores valores do índice $\mathrm{U}^{\mathrm{K}}{ }_{37}$ ' (Eq. 2) eram encontrados em baixas latitudes, regiões mais quentes; enquanto que as amostras sedimentares provenientes de altas latitudes, regiões mais frias, apresentavam valores de $\mathrm{U}^{\mathrm{K}}{ }_{37}$ bem menores. Os valores na tabela 2 exemplificam esta variação do $\mathrm{U}^{\mathrm{K}}{ }_{37}$ ' com a latitude para algumas amostras de sedimentos de superfície $(0-10 \mathrm{~cm})$ coletadas no oceano Atlântico Norte. A divulgação deste índice teve uma enorme repercussão dentro da comunidade geoquímica, pois marcava o início do uso das alquenonas como uma ferramenta molecular para a avaliação de mudanças climáticas no passado, paleoclimas, através de testemunhos marinhos.

\section{ANÁliSE, CARACTERIZAÇÃO E ESTABILIDADE RELATIVA DAS ALQUENONASS}

Ao longo dos anos, diversos procedimentos vêm sendo usado para a extração e análise das alquenonas e dos alquenoatos de alquila. No entanto, tendo em vista o grande interesse de outros grupos de pesquisa em áreas afins, como geólogos, biólogos marinhos, geofísicos e paleontólogos, de obter informações rápidas aplicando-se rotinas de extração e análise simplificadas, tornou-se necessário elaborar uma metodologia que pudesse ser relativamente simples e de boa reprodutibilida$\mathrm{de}^{16,17}$. É importante ressaltar que são necessárias, muitas vezes, centenas de amostras para se reconstruir, com alta resolução, mudanças paleoclimáticas durante algumas centenas de milhares de anos. Portanto, todo o procedimento tem que ser o mais automatizado possível. A quantidade de sedimento seco geralmente usada na extração pode variar de 1 a 6 g, dependendo do teor de carbono orgânico presente na amostra. As análises quantitativas podem ser realizadas por cromatografia gasosa de alta resolução. De acordo com o método descrito por nós ${ }^{16}$, é possível se obter uma boa separação das alquenonas e dos alquenoatos de alquila usando uma coluna de baixa polaridade, como a CPSil5CB (Chrompack, Fig. 2). O índice $\mathrm{U}^{\mathrm{K}}{ }_{37}$ ' é obtido diretamente a partir da razão das áreas dos picos das alquenonas. Algumas vezes, o teor de matéria orgânica é tão baixo (< $0,5 \%)$ que exige uma análise mais sofisticada. Para esses casos, Rosell-Melé e colaboradores ${ }^{17}$ desenvolveram, recentemente, uma rotina de análise com o uso da técnica de cromatografia gasosa acoplada à espectrometria de massas, usando amô- 
Tabela 2. Códigos e locais de algumas amostras sedimentares de superfície $(0-10 \mathrm{~cm})$ coletadas no Atlântico Norte (ver mapa na Fig. 4), com os valores dos $\mathrm{U}^{\mathrm{K}}{ }_{37}$, as temperaturas da superfície da água do mar (TSM) estimadas com o uso de diferentes equações propostas na literatura e os respectivos valores medidos diretamente na água do mar, em cada local.

\begin{tabular}{|c|c|c|c|c|c|c|}
\hline Código $^{\mathrm{a}}$ & Lat $(\mathrm{N})$ & Long $(\mathrm{O})$ & $\mathrm{U}^{\mathrm{K}}{ }_{37}{ }^{\prime}$ & $\mathrm{TSM}\left({ }^{\mathrm{o}} \mathrm{C}\right)^{\mathrm{b}}$ & $\operatorname{TSM}\left({ }^{\mathrm{o}} \mathrm{C}\right)^{\mathrm{c}}$ & $\begin{array}{c}\text { Temp.medida } \\
\left({ }^{\mathrm{d}} \mathrm{C}\right)\end{array}$ \\
\hline $61 \mathrm{~N}$ & $61^{\circ} 07^{\prime}$ & $19^{\circ} 37^{\prime}$ & 0,34 & 10,4 & 8,8 & 10,0 \\
\hline $59 \mathrm{~N}$ & $59^{\circ} 01^{\prime}$ & $21^{\circ} 49^{\prime}$ & 0,40 & 10,6 & 10,7 & 10,0 \\
\hline $48 \mathrm{~N}$ & $48^{\circ} 23^{\prime}$ & $17^{\circ} 25^{\prime}$ & 0,54 & 14,8 & 14,7 & 15,0 \\
\hline T88 & $48^{\circ} 23^{\prime}$ & $25^{\circ} 05^{\prime}$ & 0,55 & - & 15,0 & 15,0 \\
\hline $32 \mathrm{~N}$ & $32^{\circ} 52^{\prime}$ & $19^{\circ} 12^{\prime}$ & 0,62 & 19,0 & 17,0 & 20,2 \\
\hline $18 \mathrm{~N}$ & $18^{\circ} 59^{\prime}$ & $20^{\circ} 09^{\prime}$ & 0,72 & 20,0 & 20,7 & 21,8 \\
\hline
\end{tabular}

${ }^{a}$ Dados das referências $16(61 \mathrm{~N}, 59 \mathrm{~N}, 48 \mathrm{~N}, 32 \mathrm{~N}$ e $18 \mathrm{~N})$ e $40(\mathrm{~T} 88)$.

${ }^{\mathrm{b}} \mathrm{TSM}=17,35-25,12\left(\mathrm{U}^{\mathrm{K}}{ }_{37}{ }^{\prime}\right)-26,73\left(\mathrm{AA}_{36}\right)+93,90\left(\mathrm{U}^{\mathrm{K}}{ }_{37}{ }^{\prime}\right)\left(\mathrm{AA}_{36}\right)^{19}$. Equação usada para $61 \mathrm{~N}$ e $59 \mathrm{~N}$.

${ }^{\mathrm{b}} \mathrm{TSM}=3,73+11,33\left(\mathrm{U}^{\mathrm{K}}{ }_{38 \mathrm{Me}}\right)+10,86\left(\mathrm{AA}_{36}\right)+5,21\left(\mathrm{U}^{\mathrm{K}}{ }_{37}\right)^{20}$. Equação usada para $48 \mathrm{~N}, 32 \mathrm{~N}$ e $18 \mathrm{~N}$.

${ }^{\mathrm{c}} \mathrm{TSM}=\left(\mathrm{U}_{37}^{\mathrm{K}}-0,039\right) / 0,034^{33}$.

$\mathrm{U}^{\mathrm{K}}{ }_{37}^{\prime}=\left(\left[\mathrm{C}_{37: 2 \mathrm{Me}}\right] /\left[\mathrm{C}_{37: 2 \mathrm{Me}}+\mathrm{C}_{37: 3 \mathrm{Me}}\right]\right) ; \quad \mathrm{U}^{\mathrm{K}}{ }_{38 \mathrm{Me}}=\left(\left[\mathrm{C}_{38: 2 \mathrm{Me}}\right] /\left[\mathrm{C}_{38: 2 \mathrm{Me}}+\mathrm{C}_{38: 3 \mathrm{Me}}\right]\right)^{19}$.

$\mathrm{AA}_{36}=\left(\left[\mathrm{C}_{38: 3 \mathrm{Et}}\right] /\left[\mathrm{C}_{38: 3 \mathrm{Et}}\right]+\left[\mathrm{C}_{36: 3 \mathrm{FAME}}\right]+\left[\mathrm{C}_{36: 2 \mathrm{FAME}}\right]\right)^{19}$. FAME (do inglês Fatty Acid Methyl Ester).

${ }^{\mathrm{d}}$ Valores de acordo com as referências 23 (para $61 \mathrm{~N}, 59 \mathrm{~N}, 48 \mathrm{~N}$ e T88) e 65 (para $32 \mathrm{~N}$ e $18 \mathrm{~N}$ ).

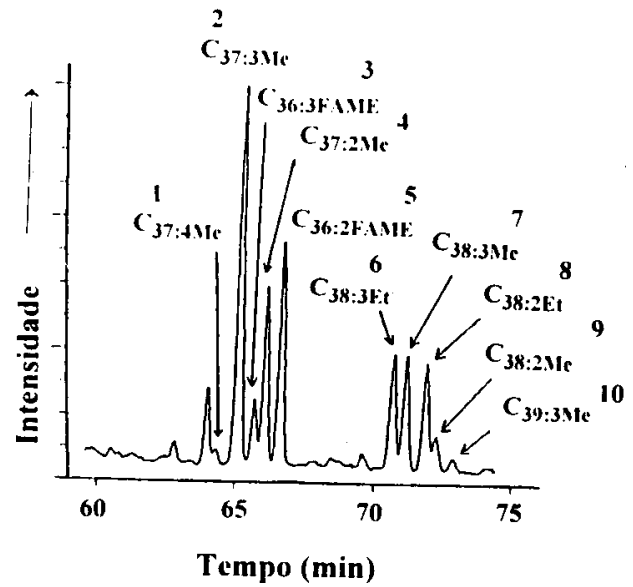

Figura 2. Cromatograma parcial do extrato orgânico total de uma amostra de sedimento de superfície $(0-3 \mathrm{~mm})$ proveniente do testemunho $61 \mathrm{~N}$ (ver código na Tabela 2). As identificações dos números e abreviaturas nos picos encontram-se na Tabela 1. Ver na referência 16 o método de extração e as condições de análise.

nia para a ionização química. Através desta técnica, o índice $\mathrm{U}^{\mathrm{K}}{ }_{37}$ 'é obtido com base nas áreas dos picos dos íons pseudomoleculares das alquenonas $\left(\mathrm{M}+\mathrm{NH}_{4}\right)^{+}$. É interessante se notar a diferença na intensidade do íon molecular de uma alquenona quando o espectro de massas é obtido pelo método Impacto de Elétrons (Fig. 3A), onde a intensidade relativa do pico $\mathrm{M}^{+}$é cerca de $3-4 \%$ da corrente iônica total, e quando se usa a Ionização Química, onde o íon $\left(\mathrm{M}+\mathrm{NH}_{4}\right)^{+}$torna-se, então, o pico base (Fig. 3B).

As estruturas das alquenonas só foram confirmadas em 1987, por Rechka e Maxwell ${ }^{18}$, através da comparação com padrões sintéticos de configurações $\mathrm{Z}$ e E. Esses autores mostraram que os componentes naturais encontrados tanto na alga Emiliania huxleyi, ou em outras espécies, quanto nos sedimentos marinhos e particulados suspensos na coluna aquática, tinham a inusitada configuração E (Fig. 1 e Tabela 1).

Após a identificação e a caracterização das alquenonas, alguns pesquisadores começaram a examinar a estabilidade relativa dos $\mathrm{C}_{37}$ di-, tri- e tetra-insaturado ${ }^{16,19}$. Esses grupos estavam interessados em verificar se o valor do índice $\mathrm{U}^{\mathrm{K}}{ }_{37}$, ou $\mathrm{U}^{\mathrm{K}}{ }_{37}$, poderia sofrer alterações desde a produção das alquenonas pelas algas passando, em seguida, pela coluna aquática, com assimilação pelo zooplâncton, até a incorporação no sedimento. Com este objetivo, foram analisadas várias amostras de águas obtidas de diversas regiões e profundidades no oceano Atlânti-

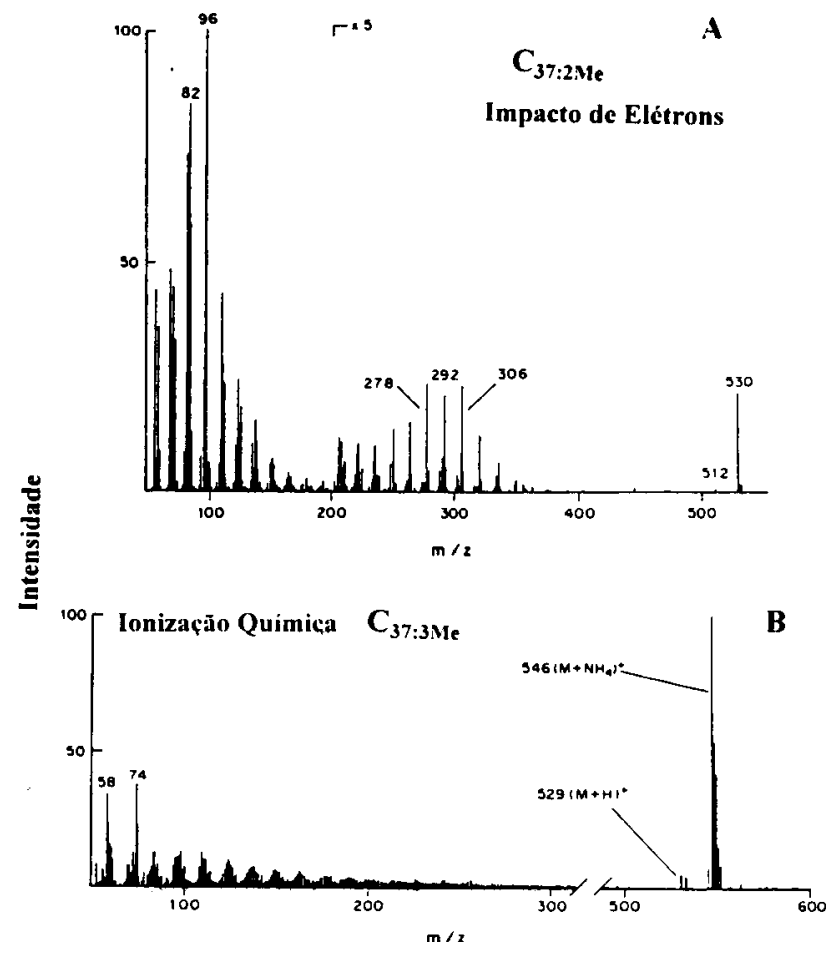

Figura 3. Espectros de massas de alquenonas sintetizadas com configurações $Z$ (ver referência 18). (A) $C_{37: 2 M e}$, analisado por Impacto de Elétrons $(70 \mathrm{eV})$ e $(\mathrm{B}) \mathrm{C}_{37: 3 \mathrm{Me}}$, analisado com Ionização Química $\left(\mathrm{NH}_{3}\right)$.

co Norte. Os valores dos $\mathrm{U}^{\mathrm{K}}{ }_{37}$ ' obtidos a partir dos particulados suspensos foram comparados com os medidos nos sedimentos depositados nas mesmas regiões do oceano. Os resultados desses estudos mostraram que para uma certa região, o valor do $\mathrm{U}^{\mathrm{K}}{ }_{37}$ ' medido no fitoplâncton permaneceu invariavelmente o mesmo que fora encontrado na coluna aquática e no sedimento $^{19}$. É importante salientar que esses trabalhos com particulados foram realizados, na grande maioria, durante a primavera $^{20}$, ou seja, no período quando ocorre o florescimento (do inglês bloom) das algas, ocasionando um grande aumento na produção primária no oceano Atlântico Norte. Para se ter uma idéia da variação média na produtividade primária, a concentração de células dessas algas na superfície do mar (aproximadamente $4 \mathrm{~m}$ de profundidade), antes e durante o florescimento, pode variar, por exemplo, de 10 a 2800 células por $\mathrm{mL}$, conforme relatado por Conte e colaboradores ${ }^{21}$. Neste mesmo 
trabalho, esses autores mostraram que a soma das concentrações das alquenonas mais os alquenoatos de alquila pode ser correlacionada à concentração de células da alga E. huxleyi (mais a Geophyrocapsa ssp.). Durante o período transitório de rápido florescimento, que pode durar alguns dias ${ }^{22,23}$, uma grande quantidade de matéria orgânica é transportada através da coluna aquática para o fundo do oceano sob a forma de macroagregados ${ }^{24,25}$, entre outras ${ }^{24}$. A deposição dos fitodetritos é sazonal ${ }^{26}$, dependendo inteiramente do material procedente da zona eufótica. Portanto, é plausível se imaginar que o valor do $\mathrm{U}^{\mathrm{K}}{ }_{37}$ fossilizado no sedimento pelas alquenonas corresponda à temperatura média da primavera durante o período de florescimento das algas (Tabela 2). Apesar desta aparente simplificada correlação entre o sinal na superfície e no sedimento, há controvérsias quanto ao valor do índice $\mathrm{U}^{\mathrm{K}}{ }_{37}$ que é de fato registrado no sedimento 27 .

Apesar das controvérsias quanto ao sinal que chega ao sedimento, se é especificamente uma resposta de uma deposição sazonal ou uma média do material que é depositado ao longo de todo o ano, não há dúvidas quanto à estabilidade relativa das alquenonas. Conforme mencionado no parágrafo anterior, as perdas das alquenonas durante a passagem pela coluna aquática são consideráveis, embora o padrão de insaturação permaneça invariável, ou seja, não existem perdas relativas ${ }^{19}$. Prahl e colaboradore $^{28}$ demonstraram que perdas das alquenonas devido a processos de oxidação em sedimentos não tiveram praticamente nenhum efeito no padrão de insaturação. Essa resistência a mudanças com a deposição também foi observada por Sikes e colaboradores ${ }^{29}$ que, inclusive, realizaram vários testes para avaliar a estabilidade desses biomarcadores. Mais recentemente, nós também demonstramos que o índice $\mathrm{U}_{37}^{\mathrm{K}}$, permanecia invariavelmente o mesmo ao longo dos primeiros $10 \mathrm{~cm}$ de profundidade em sedimentos de diversos testemunhos coletados no Atlântico Norte, apesar de ocorrerem degradações desses e de outros biomarcadores, devido à diagênese, principalmente dentro do primeiro centímetro de profundida$\mathrm{de}^{16}$. Outros trabalhos em laboratório também demonstraram a consistência do índice $\mathrm{U}^{\mathrm{K}}{ }_{37}$ ' após a remoção das alquenonas de substratos que foram submetidos a diversos processos de degradação ${ }^{30}$.

\section{RELAÇÃO ENTRE O ÍNDICE $\mathrm{U}^{\mathrm{K}}{ }_{37}$ E A TEMPERATURA DA SUPERFÍCIE DO MAR (TSM)}

Graças à variação linear do índice $\mathrm{U}^{\mathrm{K}}{ }_{37}$ ' com a temperatura da superfície da água do mar (TSM, do inglês Sea Surface Temperature - SST), isto é, a região onde as algas produzem as alquenonas, foi possível se estabelecer uma relação entre o $\mathrm{U}^{\mathrm{K}}{ }_{37}$ ' e a TSM. A maneira com que as equações propostas na literatura para esta relação são elaboradas, baseia-se fundamentalmente em três tipos de considerações: a) crescimento de culturas da alga Emiliania huxleyi ${ }^{31-33}$ e várias outras espécies (Isochrysis galbana ${ }^{31}$, Chrysotila lamellosa ${ }^{31}$, Geophyrocapsa oceanic $^{34}$ ) em laboratório a diferentes temperaturas; b) o uso de particulado suspenso na coluna aquática de diversas regiões dos oceanos Atlântico, Pacífico e Ártico e do Mar Negro $19,32,33,35,36$; c) extrato de sedimentos de superfície do oceano Pacífico ${ }^{33,35,37,38}$, do leste do Atlântico Norte ${ }^{19,27}$ e de outras regiões ${ }^{29}$. A tabela 2 contém alguns exemplos de TSM estimados com o uso do índice $\mathrm{U}^{\mathrm{K}}{ }_{37}$ no Atlântico Norte. Ainda nesta tabela, encontram-se as respectivas temperaturas da superfície da água do mar para essas mesmas regiões e que foram medidas durante outras expedições oceanográficas.

Com relação às equações usadas, nota-se, em alguns casos, o aparecimento de outros dois índices: o $\mathrm{AA}_{36}$, baseado nas abundâncias relativas dos alquenoatos de metila $\left(\mathrm{C}_{36 \text { :2FAME }} \mathrm{e}\right.$ $\left.\mathrm{C}_{\text {36:2FAME }}\right)$ e da etil-alquenona tri-insaturada $\left(\mathrm{C}_{38: 3 \mathrm{Et}}\right)$; e o $\mathrm{U}^{\mathrm{K}}{ }_{38 \mathrm{Me}}$, baseado nas abundâncias das metil-alquenonas di- e tri-insaturada $\left(\mathrm{C}_{38: 2 \mathrm{Me}}\right.$ e $\left.\mathrm{C}_{38: 3 \mathrm{Me}}\right)$ (Tabela 2). Esses dois índices mostraram-se particularmente úteis para uma equação desenvolvida a partir de amostras de particulados suspensos presentes em águas com temperaturas abaixo de $16^{\circ} \mathrm{C}\left(\mathrm{r}^{2}=0,81\right)$, no leste do Atlântico Norte. No recente trabalho de Rosell-Melé e colaboradores $^{27}$, é feita uma abordagem crítica das equações existentes para a relação $\mathrm{U}^{\mathrm{K}}{ }_{37} \times \mathrm{x}$ TSM. Os autores também propõem novas equações baseadas em um grande número de amostras de sedimentos de superfície do Atlântico Norte.

\section{APLICAÇÃO DO U ${ }^{\mathrm{K}}{ }_{37}$ : : IDENTIFICAÇÃO DOS EVENTOS DE HEINRICH}

A aplicação das alquenonas como uma ferramenta molecular para a reconstrução de paleo TSM a partir de testemunhos marinhos encontra-se amplamente difundida em todo o mundo 9,29,32,35-37,39-49. Abaixo, segue um exemplo da aplicação desses biomarcadores em um trabalho que nós realizamos com um testemunho coletado no Atlântico Norte ${ }^{40}$ (Fig. 4).

$\mathrm{O}$ testemunho T88, com aproximadamente $8 \mathrm{~m}$ de profundidade, equivalentes a cerca de 208 mil anos, convertidos para ano-calendário com ${ }^{14} \mathrm{C}$, fora anteriormente estudado por um grupo holandês ${ }^{50}$. Os resultados apresentados pelo grupo revelaram que o testemunho continha diversas informações relevantes e que poderiam ser correlacionadas com os parâmetros

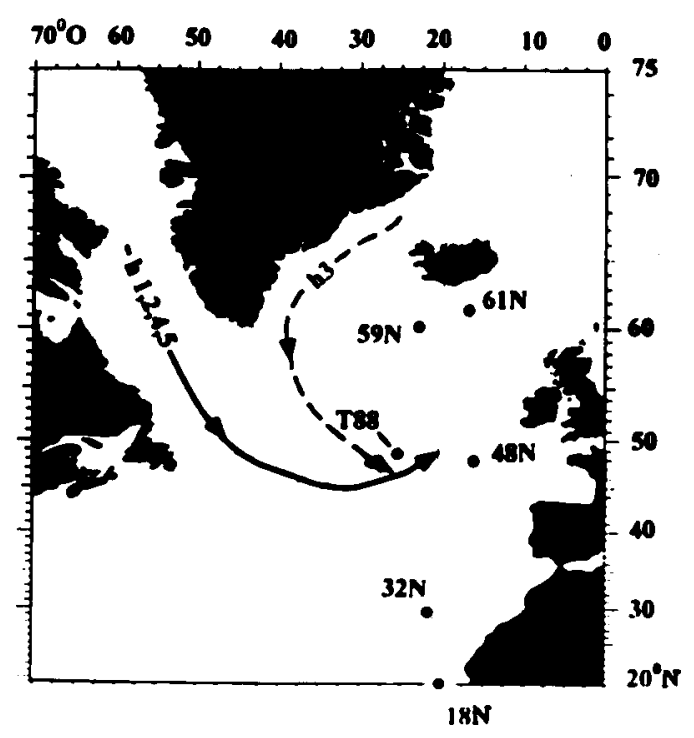

Figura 4. Mapa com as posições geográficas dos testemunhos descritos na Tabela 2. Cada um dos eventos de Heinrich, com a suposta procedência, está representado pela letra " $h$ " seguido pelo número do evento.

moleculares obtidos a partir das alquenonas. Por exemplo, através da razão isotópica de oxigênio, $\delta^{18} \mathrm{O}$ (uma medida do teor dos isótopos de oxigênio no carbonato que compõe as conchas de espécies de foraminíferas ${ }^{51}$ ), para Globigerina bulloides, e da composição de espécies de microfloras, esses autores identificaram claramente transições de intervalos glacial-interglacial (Terminações I e II há 12.300 e 130.000 anos, respectivamente) (Fig. 5A). Portanto, ao analisar as amostras de sedimentos deste testemunho, o nosso principal objetivo foi examinar variações na distribuições e abundância de biomarcadores de procedência marinha (alquenonas e alquenoatos de aquila) e terrestre (hidrocarbonetos, ácidos carboxílicos e álcoois de cadeias longas com a profundidade e correlacioná-las com as mudanças estratigráficas observadas por meio de outros parâmetros previamente analisados no testemunho. Os resultados dessas correlações são apresentados em um outro trabalho ${ }^{40}$. Um dos resul- 


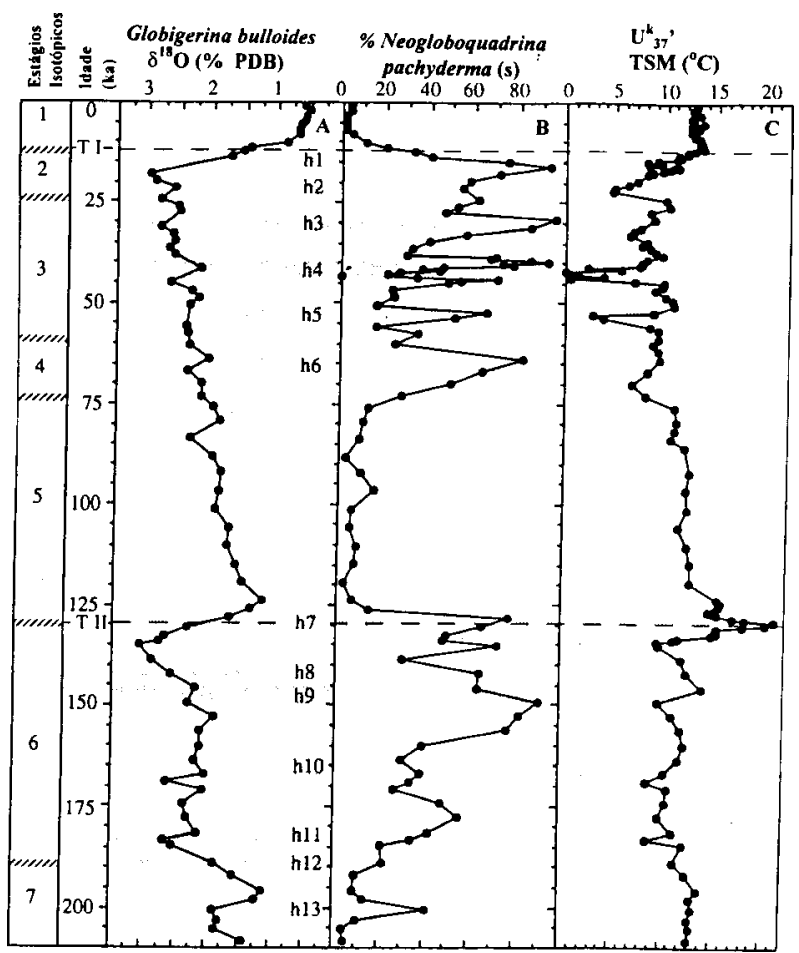

Figura 5. Comparação dos perfis sedimentológicos e dos biomarcadores ao longo do testemunho T88. (A) razão isotópica do oxigênio na foraminífera planctônica Globigerina bulloides (\% PDB, padrão), (B) $\%$ da Neogloboquadrina pachyderma sinistra e $(C)$ temperatura da superfície água do mar $\left(T S M,{ }^{\circ} C\right)$ obtida com o índice $U^{K}{ }_{37}$ através da equação proposta por Rosell-Melé e colaboradores ${ }^{27}$. Os dados dos diagramas $A$ e $B$ são da referência 50 e os do diagrama $C$ são da referência 40. Ka (quilo ano $=10^{3}$ anos). TI e TII (Terminações I e II, respectivamente) indicam as duas últimas transições glacial-interglacial.

tados mais interessantes e que será resumidamente descrito a seguir, é a identificação dos chamados eventos de Heinrich ${ }^{52}$ com o uso do $\mathrm{U}^{\mathrm{K}}{ }_{37}$.

Em 1988, Heinrich publicou que proeminentes picos com grande abundância de fragmentos líticos (detritos rochosos) espaçados de aproximadamente 10.000 anos de intervalo, haviam sido observados em uma série de três testemunhos no Atlântico Norte. Nos anos seguintes, outros autores também relataram a presença desses eventos nessa mesma região, entre as latitudes $54^{0} \mathrm{~N}$ e $41^{0} \mathrm{~N}^{53-57}$ (Fig. 4). Esses fragmentos foram depositados no fundo do oceano como consequência do transporte e derretimento de imensos icebergs. Uma análise detalhada dos detritos rochosos presentes nessas camadas, indicou que o material poderia ter mais de uma origem ${ }^{56}$. Para os cinco eventos mais recentes, quatro (h1, h2, h4 e h5) apresentaram sinais de material tipicamente encontrado na crosta ao norte de Quebec e noroeste da Groenlândia. Um dos eventos (h3) foi interpretado como procedente da região Escandinávia-IslândiaÁrtico (Fig. 4). Kreveld e colaboradores ${ }^{50}$ conseguiram identificar treze desses eventos com o testemunho T88. Com o uso do $\mathrm{U}^{\mathrm{K}}{ }_{37}$, nós conseguimos identificar onze dos treze eventos relatados por esses autores (Fig. 5C). Também usando o índice $\mathrm{U}^{\mathrm{K}}{ }_{37}$, Zhao e colaboradores ${ }^{48}$ conseguiram observar os eventos de Heinrich até mesmo em águas bem menos frias, próximas do continente africano.

No testemunho T88, o valor da TSM variou consideravelmente durante os intervalos de tempo correspondentes aos eventos de Heinrich (Fig. 5C). Embora Heinrich ${ }^{52}$ tenha reportado alguns desses eventos durante o estágio isotópico cinco, último intervalo interglacial, tanto Kreveld como nós, não identificamos nenhum desses eventos durante aquele estágio. Além da TSM, um outro resultado que reforça a visão de que as cama- das de Heinrich foram formadas durante tempos em que as águas no Atlântico Norte estavam extremamente frias, devido ao afluxo de icebergs, é o aumento na porcentagem de foraminífera Neogloboquadrina pachyderma sinistra durante os intervalos de Heinrich (Fig. 5B). Outro ponto interessante na Fig. 5 é a correlação entre a variação da TSM e a do $\delta^{18} \mathrm{O}$, medido a partir da Globigerina bulloides. Apesar de não serem muito sensíveis aos eventos de Heinrich, os valores de $\delta^{18} \mathrm{O}$ mostraram claramente as transições glacial-interglacial nas Terminações I e II. Por outro lado, através da TSM tanto os eventos de Heinrich como as duas Terminações foram observadas claramente.

\section{FUTUROS TRABALHOS}

O recente trabalho de Rosell-Melé e colaboradores ${ }^{27}$ chama a atenção para o fato de que existe uma diferença entre os valores de $\mathrm{U}^{\mathrm{K}}{ }_{37}$ obtidos por meio de culturas em laboratório e os observados na coluna aquática e no sedimento. Esses autores, baseados apenas em dados empíricos, sugerem que essa diferença seja devido a um aumento na velocidade de degradação com o número de insaturações das alquenonas, ou seja, $\mathrm{C}_{37: 4 \mathrm{Me}}>\mathrm{C}_{37: 3 \mathrm{Me}}>\mathrm{C}_{37: 2 \mathrm{Me}}$. Eles especulam ainda que essas mudanças sejam devido ao tempo de residência das alquenonas na coluna aquática, diagênese, condições de deposição, etc. No entanto, não está claro o que realmente estaria causando tal discrepância de valores. Um estudo teórico simulando diversas condições ambientais de deposição possivelmente ajudaria a entender tal comportamento.

A determinação da razão isotópica ${ }^{12} \mathrm{C}:{ }^{13} \mathrm{C}$ para biomarcadores individuais presentes em sedimento ou petróleo, encontra cada vez mais aplicação em estudos geoquímicos ${ }^{58,59}$. Esse fracionamento isotópico é de grande importância para o entendimento, por exemplo, da assimilação de carbono pelas algas ${ }^{60}$ e seria de grande interesse para as alquenonas preservadas no sedimento.

O grande número de trabalhos envolvendo a análise de alquenonas, entre outros biomarcadores, no hemisfério Norte, demonstra o interesse daqueles países em reconstruir mudanças paleoclimáticas com o objetivo de obter informações que ajudem no entendimento de mudanças climáticas futuras. $\mathrm{O}$ uso de compostos orgânicos como uma ferramenta paleoclimática é um exemplo da importância que programas multidiciplinares vêm obtendo, cada vez mais, em diversos países, o que demanda a formação de químicos com conhecimentos em áreas como a geologia e a oceanografia química, por exemplo. Em contraste com esses países do Norte, muito pouco tem sido estudado sobre a presença desses e outros biomarcadores no Atlântico Sul. Com exceção de alguns trabalhos em regiões próximas da costa (ver, por exemplo, referências 49, 61 e 62) onde existem as ressurgências (do inglês upwellings) além, é claro, da costa do Peru devido ao fenômeno $E l$ Niño ${ }^{37,63,64}$, o exame de biomarcadores que tenham implicações paleoclimáticas é praticamente inexistente nesta região do Atlântico.

\section{AGRADECIMENTOS}

Ao NERC (Natural and Environmental Research Council, Inglaterra), pela bolsa de pós-doutoramento durante o ano de 1994. Ao CNPq, pela concessão de bolsa para a realização do doutorado (1989-1993) na Unidade de Geoquímica Orgânica da Universidade de Bristol, Inglaterra.

\section{REFERÊNCIAS}

1. DSDP, do inglês Deep Sea Drilling Project. O Projeto de Perfuração em Mar Profundo era um programa científico 
internacional de expedições oceanográficas que contava com a participação de vários países. Atualmente, o programa é chamado de ODP, do inglês Ocean Drilling Project.

2. Boon, J. J.; Meer, F. W. van der; Schuyl, P. J.; de Leeuw, J. W.; Schenck, P. A.; Burlingame, A. L.; Init. Rep. Deep Sea Drilling Project. 1978, 40, 627.

3. de Leeuw, J. W.; van der Meer, F. W.; Rijpstra, W. I. C. In Advances in Organic Geochemistry; Douglas, A. G.; Maxwell, J. R., Ed.; Pergamon Press; Oxford, 1979; p 211.

4. Volkman, J. K.; Eglinton, G.; Corner, E. D. S.; Forsberg, T. E. V.; Phytochem. 1980, 19, 2619.

5. Volkman, J. K.; Eglinton, G.; Corner, E. D. S.; Sargent, J. R. In Advances in Organic Geochemistry; Douglas, A. G.; Maxwell, J. R., Ed.; Pergamon Press; Oxford, 1979; p 219.

6. Marlowe, I. T.; Green, J. C.; Neal, A. C.; Brassell S. C.; Eglinton, G.; Course, P. A.; Br. Phycol. J. 1984, 19, 203.

7. Farrimond, P.; Eglinton, G.; Brassell, S. C. In Advances in Organic Geochemistry; Leythaeuser, D.; Rullkötter, J., Ed.; Pergamon Press; Oxford, 1985; p 897.

8. Brassell, S. C.; Eglinton, G.; Marlowe, I. T.; Pflaumann, U; Sarnthein, M.; Nature 1986, 320, 129.

9. Brassell, S. C.; Brereton, R. G.; Eglinton, G.; Grimalt, J.; Liebezeit, G.; Marlowe, I. T.; Pflaumann, U.; Sarnthein, M.; Org. Geochem. 1986, 10, 649.

10. Nichols, P. D.; Johns, R. B.; Org. Geochem. 1986, 9, 25.

11. Cranwell, P. A.; Geochim. Cosmochim. Acta. 1985, 49, 1545.

12. Volkman, J. K.; Smith, D. J.; Eglinton, G.; Forsberg, T. E. V.; Corner, E. D. S.; J. Mar. Biol. Ass. U. K. 1981, 61, 509.

13. Holton, R. W.; Blecker, H. H.; Onore, M.; Phytochemistry 1964, 3, 595 .

14. Russell, N. J.; Volkman, J. K.; J. Gen. Microbiol. 1980, 118,131 .

15. Harwood, J. R.; Russell, N. J.; Lipids in Plants and Microbes; Allen, G.; Unwin, H., Ed.; London, 1984.

16. Madureira, L. A. S.; Conte, M. H.; Eglinton, G.; Paleoceanography 1995, 10, 627.

17. Rosell-Melé, A.; Carter, J. F.; Parry, A. T.; Eglinton, G.; Anal. Chem. 1995, 67, 1283.

18. Rechka, J. A.; Maxwell, J. R.; Org. Geochem. 1987, 13, 727.

19. Conte, M. H.; Eglinton, G.; Madureira, L. A. S.; Org. Geochem. 1992, 19, 287.

20. Conte, M. H.; Eglinton, G.; Deep Sea Research 1993, 40, 1935.

21. Conte, M. H.; Eglinton, G.; Madureira, L. A. S.; Philosophical Transactions: Biological Sciences 1995, 348, 169.

22. Peinert, R.; von Bodungen, B.; Smetacek, V. S. In Productivity of the Ocean: Present and Past; Berger, W. H.; Smetacek, V. S.; Wefer, G., Ed.; John Wiley \& Sons; London, 1989; p 35.

23. Weeks, A. R.; Fasham, M. J. R.; Aiken, J.; Harbour, D. S.; Read, J. F.; Bellan, I.; J. Mar. Biol. Ass. U. K. 1993, $73,253$.

24. Lee, C.; Wakeham, S. G.; Mar. Chem. 1992, 39, 95.

25. McCave, I. N.; Deep Sea Res. 1984, 22, 491.

26. Thiel, H.; Pfannkuche, O.; Schriever, G.; Lochte, K.; Gooday, A. J.; Hemleben, C.; Mantoura, R. F. G.; Turley, C. M.; Patching, J. W.; Rieman, F.; Biol. Oceanogr. 1988/ 89, 29, 203.

27. Rosell-Melé, A.; Eglinton, G.; Pflaumann, U.; Sarnthein M.; Geochim. Cosmochim. Acta. 1995, 59, 3099.

28. Prahl, F. G.; de Lange, G. L.; Lyle, M.; Sparrow, M. A.; Nature 1989, 341, 434.

29. Sikes, E. L.; Farrington, J. W.; Keigwin, L. D.; Earth Planet. Sci. Letters 1991, 104, 36.

30. Teece, M. A.; Maxwell, J. R.; Getliff, J. M.; Parkes, R. J.; Briggs, D. E. G.; Leftley, J. W. In Biomolecular palaeontology; Lyell Meeting Volume; Eglinton, G; Kay, R. L. F. Ed.; NERC Special Publication; London, 1994, p 5.
31. Marlowe, I. T.; Lipids as palaeoclimatic indicators. Tese de Doutorado. Univ. de Bristol, Inglaterra, 1994.

32. Prahl, F. G.; Wakeham, S. G.; Nature 1987, 320, 367.

33. Prahl, F. G.; Muehlhausen, L. A.; Zahnle, D.; Geochim. Cosmochim. Acta. 1988, 52, 2303.

34. Volkman, J. K.; Barrett, S. M.; Blackburn, S. I.; Sikes, E. L.; Geochim. Cosmochim. Acta. 1995, 59, 513.

35. Prahl, F. G.; Collier, R. B.; Dymond, J.; Lyle, M.; Sparrow, M. A.; Deep-Sea Res. 1993, 40, 2061.

36. Freeman, K. H.; Wakeham, S. G.; Org. Geochem. 1992, 19, 277.

37. McCaffrey, M. A.; Farrington, J. W.; Repeta, D. J.; Geochim. Cosmochim. Acta. 1990, 54, 1671.

38. Kennedy, J. A.; Brassell, S. C.; Nature 1992, 357, 62.

39. Marlowe, I. T.; Brassell, S. C.; Eglinton, G.; Green,. J. C.; Chem. Geol. 1990, 88, 349.

40. Madureira, L. A. S.; van Kreveld, S. A.; Eglinton, G.; Conte, M. H.; Ganssen, G.; van Hinte, J. E.; Ottens, J.; Paleoceanography 1997, 12, 15.

41. Rosell-Melé, A.; Eglinton, G.; Grimalt, J.; In Long-Term Climatic Variations-Data and Modelling. NATO ASI Series; Duplessy, J. -C.; Spyridakis, M. -T., Ed.; SpringerVerlag; Berlin, 1994; p 239.

42. Eglinton, G.; Bradshaw, S. A.; Rosell-Melé, A.; Sarnthein, M.; Pflaumann, U.; Tiedemann, R.; Nature 1992, 356, 423.

43. Brassell, S. C. In Organic Geochemistry: Principles and Applications; Engel, M. H.; Macko, S. A., Ed.; Plenum Press, 1993; p 699.

44. ten Haven, H. L.; Kroon, H.; Proceedings of the Ocean Drilling Program, Scientific Results 1990, 117, 445.

45. Schneider, R. R.; Müller, P. J.; Ruhland, G.; Paleoceanography 1997, 12, 36.

46. Frauke, R.; Ruhland, G.; Franck, C. B.; Müller, P. J.; Labeyrie, L. D.; Lancelot, Y.; Bard, E.; Nature 1993, 364, 319.

47. Zhao, M.; Rosell-Melé, A.; Eglinton, G.; Palaeogeogr. Palaeoclimatol. Palaeoecol. 1993, 103, 57.

48. Zhao, M.; Beveridge, N. A. S.; Shackleton, N. J.; Sarnthein, M.; Eglinton, G.; Paleoceanography. 1995, 10, 661.

49. Müller, P. J.; Schneider, R.; Ruhland, G. In Carbon Cycling in the Glacial Ocean: Constraints on the Ocean's Role in Global Change. NATO ASI Series; Zahn, R., Ed.; Springer-Verlag; Berlin, 1994; I17, p 343.

50. van Kreveld, S. A.; Knappertsbusch, M.; Ottens, J. J.; Ganssen, G.; van Hinte, J. E.; Mar. Geol. 1996, 131, 21.

51. Bradley, R. S.; Quaternary Paleoclimatology; Unwin Hyman Ltd; London, 1985; p 124.

52. Heinrich, H.; Quat. Res. 1988, 29, 142.

53. Bond, G.; Heinrich, H.; Broecker, W.; Labeyrie, L.; McManus, J.; Andrews, J.; Huon, S.; Jantschik, R.; Clasen, S.; Simet, C.; Tedesco, K.; Klas, M.; Bonani, G.; Ivy, S.; Nature 1992, 360, 245.

54. Bond, G.; Broecker, W.; Johnsen, S.; McManus, J.; Labeyrie, L.; Jouzel, J.; Bonani, G.; Nature 1993, 365, 143.

55. Broecker, W.; Bond, G.; Klas, M.; Clark, E.; McManus, J.; Clim. Dyn. 1992, 6, 265.

56. Grousset, F. E.; Labeyrie, L.; Sinko, J. A.; Cremer, M.; Bond, G.; Duprat, J.; Cortijo, E.; Huon, S.; Paleoceanography 1993, 8, 175.

57. Manighetti, B.; McCave, I. N.; Paleoceanography 1995, $10,627$.

58. Jasper, J. P.; Hayes, J. M.; Global Biogeochem. Cycles 1993, $7,451$.

59. Trindade, L. A.; Brassell, S. C.; Revista Latino-Americana de Geoquímica Orgânica 1995, 1, 1.

60. Thompson, P. A.; Calvert, S. E.; Limnol. Oceanogr. 1995, $40,673$.

61. Poynter, J.; Eglinton, G.; Proceedings of the Ocean Drilling Program, Scientifc Results 1990, 116, 155. 
62. Ruhland, G.; Müller, P. J.; Schneider, R.; 4th Int. Conf. Paleocenography (ICP IV). Kiel, 1992. Abstr., p 244.

63. Farrington, J. W.; Davis, A. C.; Sulanowski, J.; McCaffrey, M. A.; McCarthy, M.; Clifford, C. H.; Dickinson, P.; Volkman, J. K.; Org. Geochem. 1988; 13, 607.

64. McCaffrey, M. A.; Farrington, J. W.; Repeta, D. J.; Geo- chim. Cosmochim. Acta 1991, 55, 483.

65. Levitus, S.; Climatological Atlas of the World Ocean 1982, 13, U.S. Government Printing Office, Washington. 